\title{
Matinee des Bundespräsidenten
}

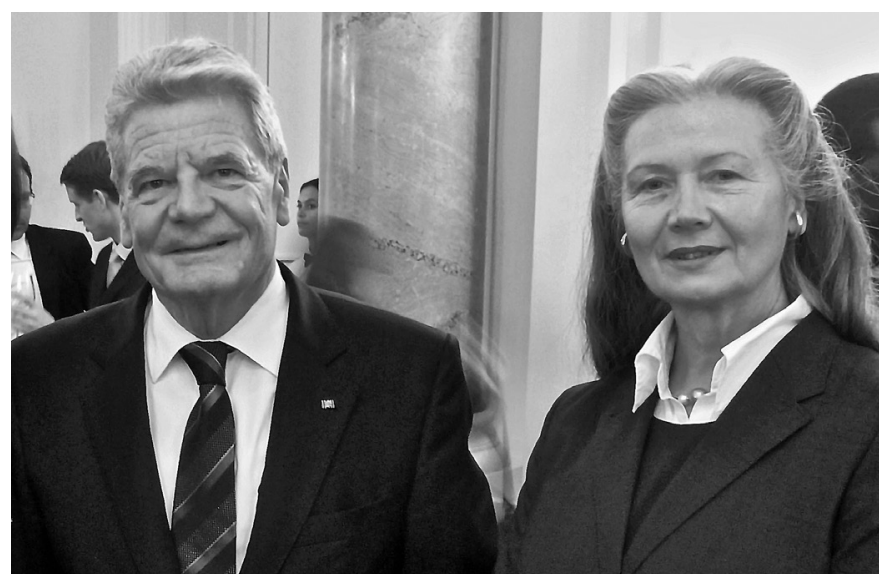

4 Bundespräsident Joachim Gauck lud aus Anlass des 65. Jahrestages der Allgemeinen Erklärung der Menschenrechte für den 6. Dezember 2013 zu einer Matinee „Menschenrechte - ein Versprechen mit Zukunft“ ins Schloss Bellevue ein. Ramona Pisal, Präsidentin des Deutschen Juristinnenbunds e.V. (djb) nahm teil.

\section{Verdienstorden für Rechtsanwältin und Notarin Jutta Wagner, Präsidentin des djb von 2005 bis 2011}

Bundespräsident Joachim Gauck hat Rechtsanwältin und Notarin Jutta Wagner das Verdienstkreuz am Bande des Verdienstordens der Bundesrepublik Deutschland verliehen. Am 10. Dezember 2014 überreichte ihr der Berliner Senator für Justiz und Verbraucherschutz Thomas Heilmann die Ordensinsignien.

Jutta Wagner, geb. 1949 in Kassel, studierte Rechtswissenschaft in Hamburg und Berlin. Nach Abschluss des Referendariats wurde sie 1978 als Rechtsanwältin in Berlin zugelassen. 1996 wurde sie zur Notarin ernannt, seit 1997 ist sie Fachanwältin für Familienrecht.

Seit langem und in vielen Bereichen engagiert sie sich ehrenamtlich, insbesondere auch seit Anfang der 90er Jahre im Deutschen Juristinnenbund e.V. (djb) und im Deutschen Anwaltverein e.V. (DAV). Unter anderem war sie von 1993 bis 2005 Ehrenamtliche Vorsitzende Richterin am Anwaltsgericht Berlin und von 1985 bis 1989 als eine der ersten Frauen Mitglied im Vorstand der Rechtsanwaltskammer Berlin.

Im djb war Jutta Wagner 1997 bis 2010 Vorstandsmitglied des djb-Landesverbands Berlin und von 1999 bis 2004 dessen Vorsitzende. Als Präsidentin des djb von 2005 bis 2011 war sie unter vielem anderen verantwortlich für den Start des vom BMFSFJ geförderten djb-Projektes „Aktionärinnen fordern Gleichberechtigung “ im Juli 2009 (http://www.djb.de/Projekt_HV/).

Sie ist Mitherausgeberin der NZFam - Neuen Zeitschrift für Familienrecht, Verlag C.H. Beck, seit 2010 Mitglied der Jury des Maria-Otto-Anwältinnen-Preises des Deutschen Anwaltvereins e.V. (DAV) und seit 2011 Mitglied der Jury des Anne-KleinFrauenpreises der Heinrich-Böll-Stiftung.

Sie unterstützt u.a. das Internationale Auschwitz Komitee, den Verein Innocence in Danger e. V., diverse Tierschutzprojekte, den Orden der Schwestern der Perpetuellen Indulgenz und die jüdische Frauenorganisation Bet Debora.
Im Folgenden ist die Rede der djb-Präsidentin Ramona Pisal anlässlich der Feier im Nordsternhaus am 10. Dezember 2014 abgedruckt:

\section{Anrede}

Es ist mir eine große Freude und eine ganz besondere Ehre, dass ich Sie heute Vormittag hier im schönen Nordsternsaal der Senatskanzlei für Justiz als Präsidentin des Deutschen Juristinnenbundes begrüßen darf. Eine große Freude, weil der Anlass für unsere Zusammenkunft ein sehr schöner und für meinen Verband ein wunderbarer Grund zum Feiern ist. Eine ganz besondere Ehre, weil Senator Heilmann mir den Vortritt lässt und weil die Frau, wegen derer wir uns hier versammelt haben, meine Vorgängerin im Amt der Präsidentin des Deutschen Juristinnenbundes ist, die mich für dieses Amt geworben, eingeführt und bis heute mit Rat und Tat begleitet hat. Der Bundespräsident hat Jutta Wagner, Rechtsanwältin und Notarin in Berlin, für ihr ehrenamtliches Engagement mit dem Verdienstkreuz der Bundesrepublik Deutschland am Bande ausgezeichnet. Das erfüllt uns mit großer Freude, Stolz und Dankbarkeit. Herzlichen Glückwunsch, liebe Jutta, zu dieser ganz besonderen Würdigung. Es bedeutet mir sehr viel, dass ich mit Ihnen dabei sein darf, wenn der Senator für Justiz gleich die Ordensinsignien an Jutta Wagner übergeben wird.

Diese Auszeichnung geht auf eine Ordensanregung des Deutschen Juristinnenbundes zurück, die Sie, Herr Senator, aufgenommen und erfolgreich auf den richtigen Weg gebracht haben. Dafür danken wir Ihnen sehr. Es ist wichtig und richtig, das ehrenamtliche Engagement von Frauen sichtbar zu machen und zu würdigen. Mit Jutta Wagner wird eine Juristin und eine Frau ausgezeichnet, die sich schon als junge Erwachsene nie nur 
für sich selbst interessiert hat. Von den Anfängen ihrer Studienzeit bis heute bringt sie sich aktiv in Gesellschaft und Politik ein. Neben einem erfolgreichen und fordernden Berufsleben als Rechtsanwältin und Notarin ist sie seit Jahrzehnten in erheblichem Umfang ehrenamtlich tätig und hat auch insoweit sehr viel erreicht. Ich weiß, liebe Jutta, wie wenig Du lange Reden und Lobpreisungen schätzt. Gleichwohl, der Anlass gebietet es, ein kleiner Überblick muss sein.

Das Studium in den frühen siebziger Jahren war bereits geprägt von vielfältigem politischem Engagement. Jutta Wagner wurde Mitglied in einem Selbstverwaltungsgremium und arbeitete dort zum Beispiel mit Jutta Limbach an Konzepten zu einer Studienreform. Sie initiierte selbst eine schnell wachsende und erfolgreiche Studentengruppe, die politisch eher links angesiedelt war, organisierte große Veranstaltungen und übte den Auftritt vor großem Publikum, durchaus mit Nutzen für den späteren Beruf. Schon als junge Rechtsanwältin wurde sie 1985 in den Vorstand der Rechtsanwaltskammer Berlin gewählt, als eine der ersten Frauen. Seit 1993 ist sie Mitglied in der Arbeitsgemeinschaft Familienrecht im Deutschen Anwaltverein, die sie mitgegründet hat, sowie der Arbeitsgemeinschaften Erbrecht und Rechtsanwältinnen im DAV. Zwölf Jahre, von 1993-2005, wirkte sie als ehrenamtliche Richterin am Anwaltsgericht Berlin, zuletzt als Vorsitzende.

Der Präsident des Deutschen Anwaltvereins, Herr Professor Ewer, und der Vorsitzende des Berliner Anwaltvereins, Herr Schellenberg, haben unsere Anregung unterstützt. Dafür mein herzlicher Dank.

Für den djb steht natürlich das ehrenamtliche Engagement von Jutta Wagner für unseren, ihren Verband im Mittelpunkt. Seit beinahe 30 Jahren ist sie Mitglied im Deutschen Juristinnenbund. 13 Jahre lang, bis 2010, war sie Mitglied im Vorstand des Landesverbandes Berlin, den sie fünf Jahre, bis 2004, als Vorsitzende geleitet und zu großer Strahlkraft geführt hat. 2005 wurde sie zur Präsidentin des djb gewählt. Dieses Amt hat sie für sechs Jahre außerordentlich erfolgreich und mit hohem persönlichem Einsatz ausgeübt. Ihre Amtszeit hat unseren Verband nachhaltig geprägt.

Viele von Ihnen wissen, und für die Politiker und Politikerinnen ist es selbstverständlich, dass Vereinsarbeit, wie Jutta Wagner sagen würde, nicht immer vergnügungssteuerpflichtig ist. Ihr besonderes Anliegen war es, in schwierigeren Zeiten einen respektvollen Umgang miteinander und eine offene und aufrichtige Kommunikationskultur zu pflegen. Dabei hat sie schmerzhafte Maßnahmen nicht gescheut, falsche Rücksichtnahmen gemieden.

Nach außen hat sie durch unermüdliche Präsenz den djb nicht nur hervorragend repräsentiert, sondern ständig weiter bekannt gemacht und vor allem für die Ziele und die Positionen unseres Verbandes in ihrer unnachahmlichen freundlich energischen Art geworben. Heutzutage finden wir uns auf Augenhöhe in der Reihe der juristischen Verbände. Zahlreiche Mitgliedsfrauen sind durch Jutta Wagner zum djb gekommen. Frauen und Männer in Zivilgesellschaft und Politik hat sie gewonnen, gemeinsam mit uns die Gleichstellung der Frauen in allen gesellschaftlichen

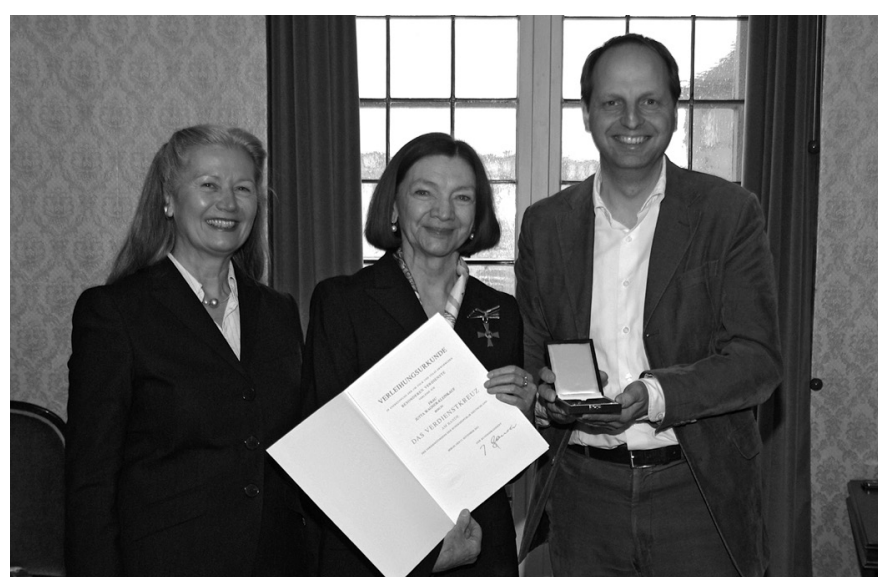

A Senator Thomas Heilmann überreicht Jutta Wagner die Ordensinsignien. Links im Bild djb-Präsidentin Ramona Pisal. Foto: Sharon Adler, Aviva-Berlin.

Bereichen aktiv mit den Mitteln des Rechts durchzusetzen. Durch ihren Einsatz und das Zusammenwirken der Kräfte, die sie für uns gebündelt hat, haben wir maßgebliche Fortschritte erzielt, die auch sichtbar sind. Als ein Beispiel möchte ich die Koalitionsvereinbarung zum Thema Frauen in Führungspositionen in der deutschen Wirtschaft nennen. Natürlich ist es nicht nur eine Frau oder nur ein Verband, die zu dieser lange überfälligen Zusicherung der künftigen Regierung geführt haben, endlich verbindliche Vorgaben für eine höhere Repräsentanz von Frauen in Führungspositionen per Gesetz festzuschreiben. Aber Jutta Wagner hat als unsere Präsidentin einen wesentlichen Beitrag dazu geleistet, dass dieses lange angestrebte rechtspolitische Ziel in greifbare Nähe rückt. Als unser Mitglied Mechtild Düsing während der Hauptversammlung der Daimler AG im Mai 2009 auf die Idee kam, den Vorstandsvorsitzenden zu fragen, warum keine Frau für die Wahl in den Aufsichtsrat vorgeschlagen wurde, tat sie das im Namen des djb, denn unsere Präsidentin Jutta Wagner hatte dem nach einem kurzen Telefonat gleich zugestimmt. In den folgenden Wochen konzipierte sie im Austausch mit dem Bundesministerium für Familie, Senioren, Frauen und Jugend anhand dieser Idee ein Projekt, das die Financial Times Deutschland später als die größte Aktion, die Deutschlands Hauptversammlungen je erlebt haben, bezeichnete. Das Projekt ist nun abgeschlossen, wir haben die Ergebnisse am 28. November 2013 vorgestellt. Mehr als 300 Hauptversammlungen haben wir in vier Jahren besucht, auch Jutta Wagner hat vor nicht immer begeistertem Publikum nach den Frauen in den Unternehmen gefragt. Mit dem Projekt „Aktionärinnen fordern Gleichberechtigung “ hat Jutta Wagner den Anstoß dafür gegeben, dass sämtliche DAX Unternehmen und 45 weitere große börsennotierte Unternehmen sich heute mit den Frauen unter ihren Beschäftigten und mit deren Karriereentwicklung ernsthaft auseinandersetzen. Und das müssen sie auch, denn es wird ein Gesetz geben, in dessen Entstehungsgeschichte Jutta Wagner mit dem maßgeblich von ihr unterstützten Projekt ein wichtiges Kapitel mitgeschrieben hat. Dafür, liebe Jutta, ein ganz herzlicher Dank. Das BMFSFJ hat das Projekt vier Jahre lang gefördert und ebenfalls diese Ordensanregung unterstützt. Auch dafür ein herzlicher Dank. 
Ein Ehrenamt kostet viel Zeit, Kraft und auch Geld. Jutta Wagner ist selbstständig. Ihre Kanzlei konnte sie als Verbandspräsidentin nicht mit voller Kraft alleine weiterführen, es mussten Angestellte bezahlt werden. In einem Interview hat sie gesagt: „Ich hatte keine realistischen Vorstellungen. Ich habe nicht im Geringsten für möglich gehalten, welchen Einsatz dieses Amt erfordert, (...) Dieses Amt ergreift einen als ganze Person. Es bestimmt für die Jahre, die man es ausübt, das Leben. Das Bedürfnis, alles hinzuwerfen, hatte ich kein einziges Mal. (...) Ich gehöre eher zu den Menschen, die, wenn sie irgendwas sehen, fragen: »Was kann ich tun? «".

Liebe Jutta, Du hast sehr viel getan, und tust es noch, nicht nur für den djb, nicht nur für Frauen. Wir freuen uns sehr, dass unsere Anregung aufgenommen worden und Dir die höchste Auszeichnung verliehen worden ist, die unser Land zu vergeben hat.

In der Amtszeit von Bundespräsident Karl Carstens (19791984) lag der Anteil an Frauen unter den jährlich verliehenen Verdienstorden mit rund 14 Prozent deutlich unter dem Bevölkerungsanteil. Dies veranlasste ihn im Jahr 1983, in die Neufassung der Ausführungsbestimmungen zum Statut des Verdienstordens hineinzuschreiben, dass „Verdiensten bei Tätigkeiten, die nach der Lebenserfahrung vor allem von Frauen ausgeübt werden, besondere Beachtung zu schenken ist“.

Im Oktober 2006 führte der Altbundespräsident Horst Köhler zusätzlich eine Quotenregelung für Frauen von mindestens 30 Prozent ein. Diese Quote wurde 2007 mit 30,3 Prozent erstmals erreicht und in den folgenden Jahren (2008: 31,2 Prozent; 2009: 30,5 Prozent) leicht überschritten. Die Vorschlagslisten der Mi- nisterpräsidenten der Bundesländer, über die die Ordensanträge vorwiegend beim Bundespräsidialamt eingereicht werden, werden nur noch angenommen, wenn von zehn Personen, die mit dem Verdienstorden ausgezeichnet werden sollen, mindestens drei Frauen sind. Das klingt vertraut: ab 2016 müssen Neubesetzungen für Aufsichtsräte in definierten Unternehmen zu 30 Prozent mit Frauen erfolgen.

Am Freitag war ich zum ersten Mal eingeladen ins Schloss Bellevue zu einer Matinee beim Bundespräsidenten aus Anlass des 65. Jahrestages der Allgemeinen Erklärung der Menschenrechte, natürlich in meiner Eigenschaft als Präsidentin des djb. Die Gelegenheit habe ich gerne ergriffen, um mich für die Verleihung des Bundesverdienstkreuzes an meine Amtsvorgängerin zu bedanken. Der Herr Bundespräsident hat mir aufgetragen, Dir, liebe Jutta, heute seine persönlichen Glückwünsche zu übermitteln und Dir herzlich zu gratulieren, was ich hiermit sehr gerne tue. Und dann hat noch irgendjemand etwas gesagt von einem old boys network, das sich immer nur gegenseitig vorschlägt und darum vermehrt Frauen in den Blick genommen werden müssen. Aber ich weiß nicht mehr, wer das war, und eine NGO mit diesem Namen kenne ich auch nicht.

Liebe Jutta, im Namen des Herrn Bundespräsidenten, aller Kolleginnen im djb und ganz persönlich gratuliere ich Dir sehr herzlich zur Verleihung des Verdienstordens der Bundesrepublik Deutschland. Wir sind sehr stolz auf Dich.

Sei Du es auch und trage ihn auf stolzgeschwellter Brust. Du hast es verdient.

\section{Der djb gratuliert}

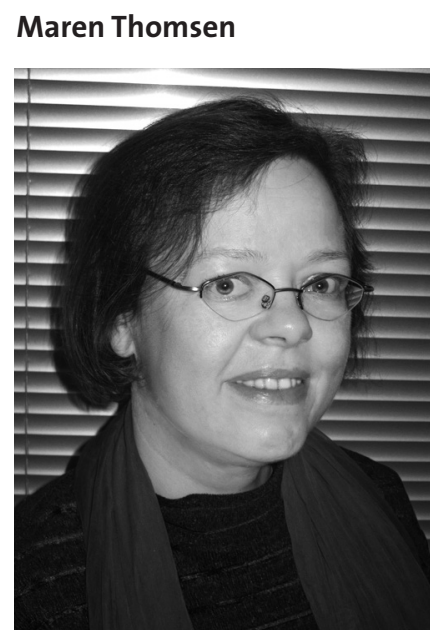

A Maren Thomsen, Präsidentin des Schleswig-Holsteinischen Oberverwaltungsgerichts. zur Ernennung als Präsidentin des Schleswig-Holsteinischen Oberverwaltungsgerichts am 6. Januar 2014. Richterin am Bundesverwaltungsgericht Maren Thomsen erhielt die Ernennungsurkunde aus den Händen der Ministerin für Justiz, Kultur und Europa des Landes Schleswig-Holstein Anke Spoorendonk, im Beisein des Staatssekretärs Herrn Dr. Eberhard Schmidt-Elsaeßer. Gleichzeitig schied sie als Richterin am Bundesverwaltungsgericht aus. Der Kieler Landtag hatte sich am 20. November

2013 einstimmig für Maren Thomsen als Nachfolgerin von Hans-Joachim Schmalz entschieden. Für die Wahl der OVGPräsidentin war eine Zwei-Drittel-Mehrheit erforderlich.
Maren Thomsen wurde 1961 in Kiel geboren. Nach Abschluss der juristischen Ausbildung begann sie ihre richterliche Laufbahn am Verwaltungsgericht Schleswig. Es folgten mehrjährige Abordnungen an das Justizministerium des Landes Schleswig-Holstein sowie als wissenschaftliche Mitarbeiterin an das Bundesverfassungsgericht. Weiteren Abordnungen an das Sozialgericht Kiel sowie das Oberverwaltungsgericht Schleswig-Holstein schloss sich im August 2005 ihre Beförderung zur Vorsitzenden Richterin am Verwaltungsgericht an. Im Februar 2007 wurde Frau Thomsen zur Vizepräsidentin des Verwaltungsgerichts Schleswig ernannt. Mit ihrer Ernennung zur Richterin am Bundesverwaltungsgericht im Juni 2007 gehörte Frau Thomsen dem u.a. für das öffentliche Dienstrecht zuständigen 2. Revisionssenat an.

Seit Mai 2008 ist sie zudem Richterin am Landesverfassungsgericht Schleswig-Holstein.

Sie ist seit 1997 djb-Mitglied. Von 1997 bis 2001 leitete sie die Kommission Migrantinnen, von 2001 bis 2005 gehörte sie als Vorsitzende der Kommission Öffentliches Recht, Europaund Völkerrecht dem Bundesvorstand an. Die Arbeit dieser Kommission gestaltet sie seitdem als aktives Mitglied mit. 